\title{
PREDACION DE SEMILLAS DE AMARANTHUS QUITENSIS H.B.K. EN UN CULTIVO DE SOJA: INFLUENCIA DEL SISTEMA DE SIEMBRA ${ }^{1}$
}

\author{
LUISA NISENSOHN, DELMA FACCINI², GUILLERMO MONTERO y MARCELA LIETTII ${ }^{3}$
}

\begin{abstract}
RESUMEN - El objetivo fue evaluar las pérdidas del banco superficial de semillas de Amaranthus quitensis H.B.K. (yuyo colorado) debidas a la predación por insectos en un cultivo de soja y en el barbecho posterior, en dos sistemas de laboreo. Los experimentos se realizaron durante las campañas 94/95 y 95/96. Para calcular la tasa de predación se emplearon bandejas cubiertas con tejido para evitar el ingreso de roedores y con tela de tul en los tratamientos testigos; en cada una se sembraron 100 semillas de la maleza y cada 15 días se registró el número de semillas remanentes. Para determinar los insectos presentes y su abundancia se emplearon trampas "pitfall". Entre los insectos capturados se encontró el carábido Notiobia cupripennis, su mayor abundancia se registró en marzo (4,5 y 5,8 insectos/trampa en convencional y 2,7 y 3,3 insectos/trampa en siembra directa), coincidiendo con las tasas de predación más altas $(5,6 \%$ y $8 \%$ en convencional y $2,7 \%$ y $3,8 \%$ en siembra directa); tanto en abundancia como en predación se observaron diferencias significativas entre ambos sistemas. A partir de este mes, las diferencias no fueron significativas, el número de insectos y la tasa de predación disminuyeron. En ambos años existió una correlación positiva entre estas variables.
\end{abstract}

Términos para índice: Notiobia cupripennis, banco de semillas, labranzas, malezas.

\section{PREDATION OF AMARANTHUS QUITENSIS H.B.K. SEEDS IN SOYBEAN CROPS: INFLUENCE OF THE TILLAGE SYSTEM}

\begin{abstract}
The objective was to evaluate the losses of the superficial bank of Amaranthus quitensis seeds, due to insect predation, in a soybean crop and in the subsequent fallow, in two tillage systems. Experiments were conducted during 1994/95 and 1995/96. To estimate predation rates, trays covered with wire meshes to prevent rodent predation, and with fine sheer net (tulle) in the control treatment were used; 100 weed seeds were sown in each tray, and the number of remaining seeds was registered every 15 days. Pitfall traps were used to identify insects species occurring in the field and to estimate their abundance. The carabid Notiobia cupripennis was captured in pitfall traps, the higher population found during March (4.5 and 5.8 insect/trap in conventional tillage and 2.7 and 3.3 insect/trap in notillage). The highest predation rates was also registered in March (5.6\% and $8 \%$ in conventional tillage and $2.7 \%$ and $3.8 \%$ in no-tillage) and both systems were significantly different as to abundance and predation. No significant differences were found from April to June, and the number of insects and predation rates decreased. There was a positive correlation between the predation rates and the values of insect abundance in both years.
\end{abstract}

Index terms: Notiobia cupripennis, seed bank, tillage, weed.

\section{INTRODUCCIÓN}

Los bancos de semillas de malezas han sido objeto de numerosos estudios debido a que desempe-

\footnotetext{
${ }^{1}$ Aceptado para publicación en 27 de julio de 1998 .

${ }^{2}$ Ing. Agr., Docente de la Cátedra de Malezas, Fac. de Ciencias Agrarias, Universidad Nacional de Rosario (UNR), C.C. no 14 (2123) Zavalla, Prov. de Santa Fe, Argentina. E-mail: dfaccini@arnet.com.ar

${ }^{3}$ Ing. Agr., Docente de la Cátedra de Zoología Agrícola, Fac. de Ciencias Agrarias, UNR. E-mail guille@tau.wamani.apc.org
}

ñan un papel crítico en el mantenimiento y restauración de sus poblaciones, especialmente en el caso de las malezas anuales en las que las semillas son el único lazo entre generaciones. Los cambios en el tamaño del banco están determinados por los ingresos, tales como lluvia de semillas, provenientes de la dispersión local o de fuentes distantes y los egresos que se producen por la germinación, la predación y la muerte fisiológica de las semillas provocada por la senescencia natural (Harper, 1977; Simpson et al., 1989). 
La predación, es entonces, uno de los procesos que puede causar pérdidas significativas de semillas. Los predadores pueden regular la densidad de semillas de algunas especies e influir en la distribución espacial (Harper, 1977; Cavers, 1983). Las pérdidas por predación influyen no sólo sobre la abundancia y distribución de las semillas en el suelo, sino sobre los estadíos siguientes del ciclo de vida. Aún cuando la magnitud de tales pérdidas sea pequeña, el impacto sobre la dinámica de las poblaciones y comunidades puede ser importante (Louda, 1989).

La mayoría de los estudios que han demostrado el efecto de la predación de semillas luego de su dispersión, sobre la estructura de una comunidad vegetal han sido realizados en habitat naturales. En estos sistemas generalmente las hormigas y los roedores son los principales predadores (Brust, 1994). Poco se conoce acerca de la actividad y el impacto de los organismos que se alimentan de semillas sobre poblaciones de malezas en ecosistemas agrícolas (Cardina et al., 1996). Algunos estudios han demostrado que entre los principales predadores granívoros en campos agrícolas se encuentran roedores, hormigas y carábidos (Brust \& House, 1988). En estudios realizados en Argentina se ha comprobado que en el caso de una maleza importante como Sorghum halepense (L.) Pers., la pérdida diaria de semillas producida por roedores varió entre $1 \%$ y $18 \%$ en el cultivo de soja temprana, y entre $3 \%$ y $15 \%$ durante el ciclo del cultivo en maíz (Scopel et al., 1988), y en áreas no cultivadas se estableció que la densidad de semillas del banco de esta especie estuvo determinada principalmente por los ingresos producidos por la lluvia de semillas y por las pérdidas causadas por los predadores de semillas (Van Esso \& Ghersa, 1989).

En los agroecosistemas las modificaciones producidas al ambiente por los distintos tipos de labranza afectan tanto a las poblaciones de malezas como a las de artrópodos. En el caso de las malezas, estas modificaciones influyen principalmente sobre la abundancia y diversidad de sus comunidades (Mohler \& Callaway, 1992), la densidad de semillas presentes en el suelo (Dyer, 1995) y su distribución en el perfil (Van Esso et al.,1986; Cavers \& Benoit, 1989). En las poblaciones de artrópodos, los distintos sistemas de labranza determinan la distribución y abundancia de los mismos (Stinner \& House, 1990) y la presencia de residuos vegetales en superficie influye marcadamente en su comportamiento, desarrollo y sobrevivencia (Musick, 1970; House \& Stinner, 1983).

Amaranthus quitensis H.B.K. es una maleza anual que causa importantes pérdidas en cultivos de verano. Densidades de sólo $1 \mathrm{pl} / \mathrm{m}^{2}$ pueden ocasionar disminuciones del $13 \%$ en el rendimiento de los cultivos de soja (Leguizamón et al., 1994). Esta maleza puede producir grandes cantidades de semillas; en cultivos de soja, que no recibieron control químico o mecánico, se han registrado valores medios de 215.000 semillas $/ \mathrm{m}^{2}$ con una viabilidad del $98 \%$. En estimaciones del banco de semillas realizados en dicha área antes de la cosecha del cultivo el número de semillas presentes fue de 33.000 por $\mathrm{m}^{2}$ (Faccini \& Nisensohn, 1994). Sin embargo, las pérdidas por germinación registradas son muy pequeñas, sólo se recluta un $4,1 \times 10^{-3}$ del total de las semillas presentes en el banco y las pérdidas por senescencia natural luego de permanecer enterradas durante 6 meses a 3 , 10 y $15 \mathrm{~cm}$ de profundidad alcanzan valores medios de 16\% (Nisensohn \& Faccini, 1993). La predación podría entonces tener un rol importante en la reducción del número de semillas de $A$. quitensis presentes en el banco.

Dellafiore \& Polop (1994) encontraron evidencias de que Calomys musculinus (Waterhouse), un roedor que posee una dieta granívora con un comportamiento oligófago, basa la misma principalmente en dos especies y una de ellas es A. quitensis. En los experimentos realizados se registró su consumo en el $87 \%$ de los individuos analizados.

Existe escasa información del papel que juegan otros predadores, como los insectos en la disminución del número de semillas de esta maleza.

El objetivo de este trabajo fue evaluar las pérdidas del banco superficial de semillas de A. quitensis ocasionadas por la predación por insectos en un cultivo de soja y en el barbecho posterior, en sistemas de siembra directa y de labranza convencional.

\section{MATERIAL Y METODOS}

Los experimentos se llevaron a cabo en la localidad de Zavalla (Santa Fe, Argentina ) a $33^{\circ} 0^{\prime}$ de latitud y $60^{\circ} 53^{\prime}$ 
de longitud en cultivos de soja durante las campañas 1994/95 y 1995/96. Un área de $5.000 \mathrm{~m}^{2}$ fue laboreada de manera convencional (la labor principal se realizó con arado de rejas) y en otra área de igual tamaño se realizó siembra directa. La variedad de soja Asgrow 6283 se sembró, en ambos años, a mediados de noviembre.

En el centro de cada una de éstas parcelas, se colocaron al azar, 20 bandejas plásticas de $18 \times 11 \times 2,5 \mathrm{~cm}, 10$ de las cuales se cubrieron con tejido de alambre que permitía el ingreso de insectos, pero excluía a los roedores y las 10 restantes se utilizaron como testigos, cubriéndolas con tela de tul que impedía tanto el acceso de roedores como de insectos. En la zona de laboreo convencional las bandejas se completaron con tierra y en la de siembra directa con tierra más rastrojo. En todos los casos, sobre la superficie de cada bandeja se sembraron 100 semillas de A. quitensis. Las panojas de las plantas de la maleza ubicadas alrededor de las bandejas se cortaron para impedir el ingreso de semillas a las mismas.

Desde mediados de marzo, momento en que comienza la dispersión de las semillas, y hasta fines de junio, aproximadamente cada 15 días las bandejas se retiraron y fueron reemplazadas por otras con el número inicial de semillas de la maleza. Las muestras se lavaron y secaron para recuperar y contar las semillas remanentes y registrar el número de semillas predadas. Con estos datos se calculó la tasa de predación según la siguiente fórmula $\mathrm{P}=100\left(1-\mathrm{r}^{1 / t}\right)$ dónde $\mathrm{P}$ es el porcentaje de semillas predadas por día, $\mathrm{r}$ es el porcentaje de semillas remanentes y t es la duración del experimento en días (Mittelbach \& Gross, 1984 ).

Para establecer la presencia y cuantificar los niveles poblacionales de insectos predadores de semillas en el suelo se registró la abundancia de insectos capturados en trampas "pitfall"; este método de muestreo tambien se utiliza como indicador de la actividad superficial de los insectos (Greenslade, 1964). En cada sistema se ubicaron a unos $10 \mathrm{~m}$ de las bandejas, 10 trampas de $12 \mathrm{~cm}$ de diámetro conteniendo $100 \mathrm{cc}$. de ácido acético al $10 \%$, las que fueron revisadas cada 15 días.

Los datos de temperatura media del aire se obtuvieron de los registros diarios de la Estación Meteorológica de la Facultad de Ciencias Agrarias de la Universidad Nacional de Rosario, ubicada a $500 \mathrm{~m}$ del experimento.

Tanto las tasas de predación como la abundancia de insectos se analizaron estadísticamente utilizando un test de comparación de medias (test de t). Previo al análisis, los datos se transformaron utilizando arc sen $(x+1)^{1 / 2}$ en el caso de las tasas de predación y $(x+1)^{1 / 2}$ para los valores de abundancia. Para describir la relación entre la tasa de predación y la abundancia de insectos se empleó el análisis de regresión.

\section{RESULTADOS Y DISCUSIÓN}

Tanto en labranza convencional como en siembra directa, en ambos años, en las bandejas utilizadas como testigos, de las que fueron excluídos roedores e insectos, no se registraron pérdidas de semillas por germinación o muerte; siempre se recuperaron las 100 semillas iniciales. En cambio, en las bandejas cubiertas con tejido, en las que los insectos podían ingresar, se observaron pérdidas de semillas. Entre los insectos capturados se encontraron 3 especies de carábidos pertenecientes a la tribu Harpalini: Polpochila flavipes Dej., Selenophorus alternans Dej. y Notiobia cupripennis Dej. que era la mas abundante y frecuente. La mayoría de las especies de carábidos son agentes naturales de control de plagas insectiles, no obstante, muchas especies de la tribu Harpalini son conocidas como predadores de semillas de malezas (Johnson \& Cameron, 1969; Best \& Beegle, 1977; Lund \& Turpin, 1977; Brust \& House, 1988). N. cupripennis es una de las especies de esta tribu común en los agroecosistemas de la pampa húmeda de la Argentina y ha sido citada como predador de semillas; fue observada por Bosq (1942) alimentándose de granos de cebada y centeno.

El efecto de la labranza y del manejo del rastrojo sobre la fauna del suelo difiere entre agroecosistemas y regiones geográficas (Mack \& Backman, 1990). Las especies de carábidos varían su respuesta a la labranza (Clark et al., 1993), se ha observado que aún la misma especie puede responder de distinta manera al mismo tratamiento de labranza, en diferentes sitios (Weiss et al., 1990). Según House y Stinner (1983) los insectos de los sistemas de labranza convencional parecen ser más móviles que aquellos de los agroecosistemas de siembra directa. Existe un gran número de experimentos en los que se determinó que hay un incremento de la abundancia de carábidos en labranza reducida (House \& All, 1981; Stinner \& House, 1990; Cardina et al., 1996). Sin embargo, en otros ensayos se registró un mayor número de carábidos en labranza convencional (Barney \& Pass, 1986; Cárcamo et al., 1995) o no se encontraron diferencias entre los sistemas (Tyler \& Ellis, 1979) como ocurre en los experimentos realizados.

Los valores de abundancia de $N$. cupripennis se observan en el Cuadro 1. En los dos años, entre el 
15 y 30 de marzo, en ambos sistemas de laboreo, coincidiendo con el período reproductivo de la especie, se capturó el número de insectos más alto, indicando una elevada actividad superficial y un mayor nivel poblacional. Sólo durante este período el número de insectos presentes en labranza convencional fue significativamente mayor que el encontrado en siembra directa. A partir del mes de abril las diferencias no fueron significativas y se observó una disminución de la abundancia; esta disminución puede relacionarse con la temperatura, que es el principal factor regulador de la actividad superficial de los carábidos (Luff, 1982). La disminución de la temperatura media desde abril $\left(10,5^{\circ} \mathrm{C}\right)$ podría restringir la actividad superficial de esta especie, hasta que finalmente en mayo o junio no se capturan más individuos en las trampas.

Las tasas de predación de las semillas de $A$. quitensis se observan en el Cuadro 2. Entre el 15 y 30 de marzo, en ambos sistemas de laboreo, coincidiendo con el momento de mayor abundancia y actividad de los insectos se registraron los valores más altos de predación de todo el período de consumo, los valores fueron de 5,6\% y $8 \%$ en labranza convencional y $2,7 \%$ y $3,8 \%$ en siembra directa para las campañas 1994/95 y 1995/96 respectivamente. Sólo en este período hubo diferencias significativas entre sistemas de labranza en los recuentos realizados. A partir de abril, cuando el número de insectos capturados fue menor, la predación disminuyó hasta cero durante el mes de junio. Según Crawley (1992) las tasas de predación pueden variar en el tiempo y en el espacio. Estas variaciones observadas a lo largo del año en la tasa de predación coinciden con lo observado en otros estudios. Cardina et al. (1996) registró, en cultivos de maíz, variaciones en el porcentaje de predación diario de semillas de Abutilon theophrasti Medicus, desde 1,2\% a 57\%.

Los valores obtenidos durante el año en los experimentos realizados son similares a los citados por Brust \& House (1988) quienes en un período de 5 semanas en el otoño, obtuvieron tasas de predación del $1 \%$ al $5 \%$ en campos laboreados y resultan inferiores a los observados en campos no perturbados en los que los valores de predación variaron entre el $1 \%$ y el 20\% diario (Mittelbach \& Gross, 1984) y en praderas dónde las pérdidas fueron superiores al $60 \%$ (Platt, 1976).

En la Fig. 1 se observan las regresiones que describen la relación entre abundancia de $N$. cupripennis y la tasa de predación de A. quitensis, estas dos variables se relacionan positivamente a través de una función lineal. En todos los casos, los coeficientes de regresión fueron significativos; en siembra convencional (Fig. 1a y 1b) los valores de $\mathrm{R}^{2}$ para la campaña 94/95 y 95/96 fueron 0,767 y 0,773 , y en siembra directa (Fig. 1c y 1d) los valores de $\mathrm{R}^{2}$ para la campaña 94/95 y 95/96 fueron 0,398 y 0,549. El menor ajuste observado en siembra directa podría explicarse por la incidencia en la tasa de predación de otro factor, como el incremento de semillas de malezas, principalmente gramíneas anuales, cerca de la superficie (Froud-Williams et al., 1984; Wrucke \& Arnold, 1985; Ball, 1992; Hurle, 1993), las que podrían comportarse como otra fuente de alimentación para los insectos. Por ejemplo Digitaria sanguinalis (L.) Scop. es una gramínea cuyas semillas, aunque son menos preferidas que $A$. quitensis (Lietti et al., 1995),

CUADRO 1. Abundancia de Notiobia cupripennis (insectos/trampa) en sistemas de labranza convencional y en siembra directa durante las campañas 1994/95 y 1995/96.

\begin{tabular}{llccccccc}
\hline Año & Labranza & \multicolumn{7}{c}{ Fechas } \\
\cline { 3 - 8 } & & $15-30 / 3$ & $1-15 / 4$ & $16-30 / 4$ & $1-15 / 5$ & $16-30 / 5$ & $1-15 / 6$ & $16-30 / 6$ \\
\hline \multirow{2}{*}{$1994 / 95$} & Laboreo convencional & $4,5^{*}$ & 1,7 & 0,3 & 0,3 & 0 & 0 & 0 \\
& Siembra directa & $2,7^{*}$ & 1,1 & 0,2 & 0,25 & 0 & 0 & 0 \\
\hline $1995 / 96$ & Laboreo convencional & $5,8^{*}$ & 2,8 & 1,5 & 1,5 & 0,7 & 0,4 & 0 \\
& Siembra directa & $3,3^{*}$ & 2,8 & 1 & 1 & 0,5 & 0,1 & 0 \\
\hline
\end{tabular}

* Diferencias significativas entre tratamientos con $\mathrm{p} \leq 0,05$ dentro de cada año. 
CUADRO 2. Tasas de predación de semillas de Amaranthus quitensis (\%) en sistemas de labranza convencional y en siembra directa durante la campaña 1994/95 y 1995/96.

\begin{tabular}{llrrrrrrr}
\hline \multirow{2}{*}{ Año } & Labranza & \multicolumn{7}{c}{ Fechas } \\
\cline { 3 - 8 } & & $15-30 / 3$ & $1-15 / 4$ & $16-30 / 4$ & $1-15 / 5$ & $16-30 / 5$ & $1-15 / 6$ & $16-30 / 6$ \\
\hline \multirow{2}{*}{$1994 / 95$} & Laboreo convencional & $5,6^{*}$ & 1,8 & 1,7 & 1 & 0,44 & 0 & 0 \\
& Siembra directa & $2,7^{*}$ & 1,2 & 1,6 & 1,3 & 0,34 & 0 & 0 \\
\hline \multirow{2}{*}{$1995 / 96$} & Laboreo convencional & $8,0^{*}$ & 4,5 & 2,3 & 3,4 & 1,9 & 2,1 & 0 \\
& Siembra directa & $3,8^{*}$ & 4,1 & 2,8 & 2,3 & 1,9 & 2,1 & 0 \\
\hline
\end{tabular}

* Diferencias significativas entre tratamientos con $\mathrm{p} \leq 0,05$ dentro de cada año.
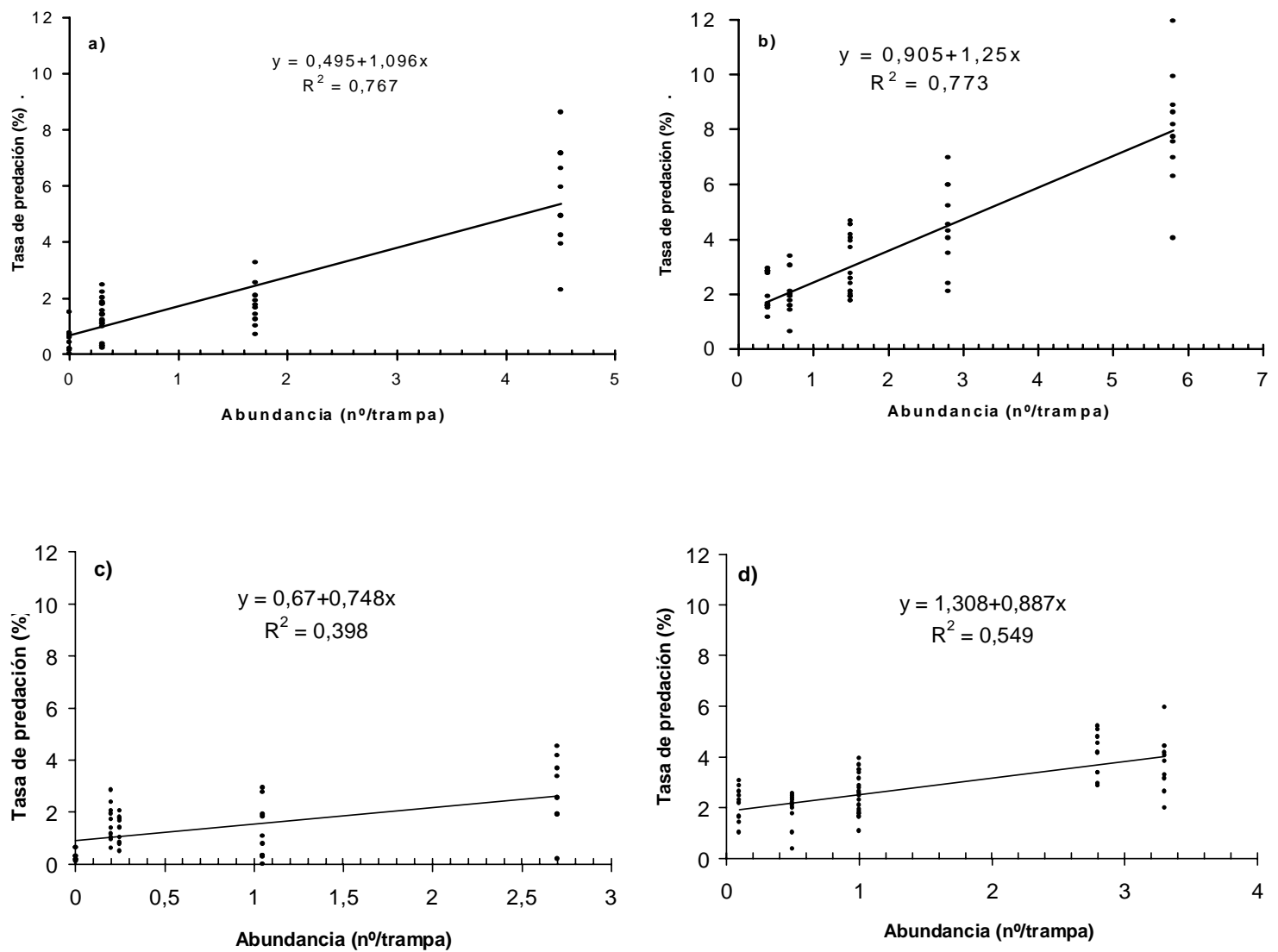

FIG. 1. Relación entre la abundancia de Notiobia cupripennis y la tasa de predación de semillas de Amaranthus quitensis en dos sistemas de laboreo: a) labranza convencional, campaña 1994/95; b) labranza convencional, campaña 1995/96; c) siembra directa, campaña 1994/95 y d) siembra directa, campaña 1995/96. 
tambien son consumidas por $N$. cupripennis, pudiendo influir su presencia en este sistema sobre las tasas de predación de $A$. quitensis.

El porcentaje total de semillas predadas sobre el de semillas ofrecidas, durante el período de consumo, fue en siembra convencional de $32 \%$ y $56 \%$ mientras que en siembra directa fue de $22 \%$ y $43 \%$ en las campañas 1994/95 y 1995/96, respectivamente. Teniendo en cuenta estos resultados, la recomendación de postergar el laboreo del suelo para prolongar el lapso durante el cual las semillas están expuestas a predación, como en el caso de Sorghum halepense (Vitta et al., 1989), tambien sería válida para favorecer la pérdidas de semillas de $A$. quitensis de la superficie del suelo.

La presencia de este predador granívoro en un agroecosistema, puede jugar un rol importante disminuyendo la densidad de semillas de $A$. quitensis del banco superficial e influyendo de esta manera sobre el establecimiento de las futuras poblaciones de plántulas de esta maleza.

\section{CONCLUSIONES}

1. La presencia del carábido N. cupripennis capaz de predar semillas de la maleza $A$. quitensis se determina en ambos sistemas de laboreo.

2. Tanto las mayores tasas de predación de semillas, como los valores más altos de abundancia del insecto se registran durante el mes de marzo.

3. El porcentaje total de predación, en promedio para ambos años, en el sistema de labranza convencional es de 43,6\% y en siembra directa es de $32,5 \%$.

\section{AGRADECIMIENTOS}

Los autores agradecen la colaboración en los análisis estadísticos de la Est. Vilma Bisaro y las sugerencias del Lic. Daniel Tuesca.

\section{REFERENCIAS}

BALL, D.A. Weed seedbank response to tillage, herbicides, and crop rotation sequence. Weed Science, v.40, p.654-659, 1992.

BARNEY, R.J.; PASS, B.C. Foraging behavior and feeding preference of ground beetles (Coleoptera: Carabidae) in Kentucky alfalfa. Journal of Economic Entomology, v.79, p.1334-1337, 1986.

BEST, R.L.; BEEGLE, C.C. Food preferences of five species of carabids commonly found in Iowa corn fields. Enviroment Entomology, v.1, n.6, p.9-12, 1977.

BOSQ, J.M. Segunda lista de coleópteros de la República Argentina dañinos a la agricultura. Ingeniería Agronómica, v.4, n.18, p.17-26, 1942.

BRUST, G.E. Seed predators reduce broadleaf weed growth and competitive ability. Agriculture, Ecosystems and Enviroment, v.48, p.27-34, 1994.

BRUST, G.E.; HOUSE, G.J. Weed seed destruction by arthropods and rodents in low-input soybean agroecosystems. American Journal of Alternative Agriculture, v.3, p.19-25, 1988.

CARCAMO, H.; NIEMALA, J.; SPENCE, J.R. Farming and ground beetles: Effects of agronomic practice on populations and community structure. The Canadian Entomologist, n.127, p.123-140, 1995.

CARDINA, J.; NORQUAY, H.M.; STINNER, B.R.; McCARTNEY, D.A. Postdispersal predation of Velvetleaf (Abutilon theophrasti) seeds. Weed Science, v.44, p.534-539, 1996.

CAVERS, P. Seed demography. Canadian Journal of Botany, v.61, p.3578-3590, 1983.

CAVERS, P.B.; BENOIT, D.L. Seed bank in arable land. In: LECK, M.A.; PARKER, V.T.; SIMPSON, R.L. (Eds.). Ecology of soil seed banks. New York: Academic Press, 1989. Ch.14, p.309-326.

CLARK, M.S.; LUNA J.M.; STONE, N.D.; YOUNGMAN, R.R. Habitat preferences of generalist predators in reduced tillage corn. Journal of Entomological Science, v.28, p.404-416, 1993.

CRAWLEY, M.J. Insect herbivores and plant population dynamics. Annual Review of Entomology, v.34, p.531-564, 1992.

DELLAFIORE, C.; POLOP, J. Feeding habitats of Calomys musculinos (Waterhouse) in the crop fields and its borders. Mastozzología Neotropical, v.1, p.45-50, 1994.

DYER, W.E. Exploiting weed seed dormancy and germination requirements through agronomic practices. Weed Science, v.43, p.498-503, 1995. 
FACCINI, D.E.; NISENSOHN, L.A. Dinámica de la población de yuyo colorado (Amaranthus quitensis H.B.K.). Influencia de los tratamientos químicos y mecánicos en un cultivo de soja. Pesquisa Agropecuaria Brasileira, v.29, n.7, p.1041-1050, jul. 1994

FROUD-WILLIAMS, R.; CHANCELLOR, P.; DRENNAN, D.S. The effects of seed burial and soil disturbance on emergence and survival of arable weeds in relation to minimal cultivation. Journal of Applied Ecology, v.21, p.629-641, 1984

GREENSLADE, P.J. Pitfall trapping as a method for studying populations of carabidae (Coleoptera). Journal of Animals Ecology, v.33, n.2, p.301-310, 1964.

HARPER, J.L. Population biology of plants. London: Academics Press, 1977. 582p.

HOUSE, G.J.; ALL, J.N. Carabids beetles in soybean agroecosystems. Environmental Entomology, v.10, p.194-196, 1981.

HOUSE, G.J.; STINNER, B.R. Arthropods in no-tillage soybean agroecosystems: community composition and ecosystem interactions. Environmental Management, v.7, n.1, p.23-28, 1983.

HURLE, K. Integrated management of grass weeds in arable crops. In: BRIGHTON CROP PROTECTION CONFERENCE WEEDS. Proceedings.. Brighton, UK: British Crop Protection Council, 1993. v. 1, p. $81-88$

JOHNSON, N.; CAMERON, R.S. Phytophagous ground beetles. Annals of the Entomological Society of America, v.62, n.4, p.909-914, 1969.

LEGUIZAMON, E.S.; FACCINI, D.E.; NISENSOHN, L.A.; PURICELLI, E.; MITIDIERI, A.; LOPEZ, J.; RAINERO, H.; RODRIGUEZ, N.; PAPA, J.C.; ROSSI, R.; CEPEDA, S.; PONSA, J.C.; MORENO, R.; FAYA, L. Funciones de daño y cálculo de pérdidas por malezas en el cultivo de soja. INTA Informe Técnico, Pergamino, n.296, p.1-19, 1994.

LOUDA, S.M. Predation in the dynamics of seed regeneration. In: LECK, M.A.; PARKER, V.T.; SIMPSON, R.L. (Eds.). Ecology of soil seed banks. New York: Academic Press, 1989. Ch.3, p.25-51.

LUFF, M.L. Population dinamics of Carabidae. Annual Applied Biology, v.101, n.1, p.164-170, 1982.
LUND, R.D.; TURPIN, F.T. Carabid damage to weed seeds found in Indiana corn field. Enviromental Entomology, v.6, p.695-698, 1977.

MACK, T.P.; BACKMAN, C.B. Effect of two planting dates and three tillage systems on the abundance of lesser cornstalk borer (Lepidoptera: Pyralidae), other selected insects, and yield in peanut fields. Journal of Economic Entomology, v.83, p.1034-1041, 1990.

MITTELBACH, G.G.; GROSS, K.L. Experimental studies of seed predation in old-fields. Oecologia, v.65, p.713, 1984.

MOHLER, C.L.; CALLAWAY, M.B. Effects of tillage and mulch on the emergence and survival of weeds in sweet corn. Journal Applied Ecology, v.29, p.2134, 1992.

MUSICK, G.J. Insect problems associated weed not till crops. Crops and Soil Magazine, v.23, p.18-19, 1970.

NISENSOHN, L.; FACCINI, D. Persistencia de semillas de yuyo colorado en un suelo sin remoción. Turrialba, v.43, n.2, p.138-142, 1993.

PLATT, W.J. The natural history of a fugitive prairie plant (Marabilis hirsuta, (Rish) Mac M). Oecologia, v.22, p.399-409, 1976.

SCOPEL, A.; BALLARE, C.; GHERSA, C.M. Role of seed reproduction in the population ecology of Sorghum halepense in maize crops. Journal of Applied Ecology, v.25, p.951-962, 1988.

SIMPSON, R.L.; LECK, M.A.; PARKER, V.T. Seed banks: general concepts and methodological issues. In: LECK, M.A.; PARKER, V.T.; SIMPSON, R.L. (Eds.). Ecology of soil seed banks. New York: Academic Press, 1989. Ch.1, p.3-8.

STINNER, B.R.; HOUSE, G.J. Arthropods and other invertebrates in conservation-tillage agriculture. Annual Review of Entomology, v.35, p.299-318, 1990.

TYLER, B.M.J.; ELLIS, C.R. Ground beetles in three tillage plots in Ontario and observations on their importance as predators of the northern corn rootworm, Diabrotica longicornis (Coleoptera: Chrysomelidae) Proceedings of the Entomological Society of Ontario, v.110, p.65-73, 1979.

VAN ESSO, M.L.; GHERSA, C.M. Dynamics of Sorghum halepense seeds in the soil of an incultivated field.

Pesq. agropec. bras., Brasília, v.34, n.3, p.377-384, mar. 1999 
Canadian Journal of Botany, v.67, p.940-944, 1989.

VAN ESSO, M.L.; GHERSA, C.M.; SORIANO, A Cultivation effects on the dynamics of a Johnsongrass seed population in the soil profile. Soil \& Tillage Research, v.6, p.325-335, 1986.

VITTA， J.I.; TUESCA， D.; PURICELLI， E.; LEGUIZAMON, E. Modelo conceptual de la dinámica del banco de semillas de Sorgo de Alepo (Sorghum halepense (L.) Pers.) en la secuencia Trigo-Soja. Implicancias en la persistencia de la maleza.
Revista de la Facultad de Agronomía de la Universidad de Buenos Aires, t.10, n.1/2, p.1115,1989

WEISS, M.; BALSBAUGH, J.R.E.; FRENCH, E.; HOAG, $B$. Influence of tillage management and cropping system on ground beetle (Coleoptera: Carabidae) fauna in the northern great plains. Environmental Entomology, v.19, p.1388-1391, 1990.

WRUCKE, M.A.; ARNOLD, W.E. Weed species distribution as influenced by tillage and herbicides. Weed Science, v.33, p.853-856, 1985. 